\title{
Formulation of the Nonabelian Tensor Square of a Bieberbach Group
}

\author{
Rohaidah Masri, Nor Fadzilah Abdul Ladi, Nor'ashiqin Mohd Idrus, Nor Haniza Sarmin
}

\begin{abstract}
A Bieberbach set can be categorized as a torsion free crystallographic set. Some properties can be explored from the set such as the property of nonabelian tensor square. The nonabelian tensor square is one type of the homological factors of sets. This paper focused on a Bieberbach set with $C_{2} \times C_{2}$ as the point set of lowest dimension three. The purpose of this paper is to determine the generalization of the formula of the nonabelian tensor square of one Bieberbach set with point set $C_{2}$ $\times C_{2}$ of lowest dimension three which is denoted by $S_{2}(3)$ up to dimensionn. The polycyclic presentation, the abelianization of $S_{2}(3)$ and the central subgroup of the nonabelian tensor square of $S_{2}(3)$ are also presented.
\end{abstract}

Keywords: Bieberbach set, Nonabelian Tensor Square, Homological Functor.

\section{INTRODUCTION}

The Bieberbach sets are also known as a torsion free crystallographic sets. The Bieberbach set satisfies the short exact sequence

$$
\longrightarrow \stackrel{\varphi}{\longrightarrow} G \stackrel{\phi}{\longrightarrow} P \longrightarrow 1,
$$

where this set is basically an extension of a free abelian set $L$ of finite rank by a finite point set $P$ such that $G / \varphi(L) \cong P$. In this paper, one Bieberbach set, which is the second set of lowest three dimension with $C_{2} \times C_{2}$ as the group point is explored.

The nonabelian tensor square is an interesting property of crystallographic set that can be revealed. Let $G$ be a set, the nonabelian tensor square of $G$, denoted by $G \otimes G$, is generated by generators $g_{1} \otimes g_{2}$, for any $g_{1}, g_{2} \in G$, satisfies the relations

$$
\begin{aligned}
& g_{1} g_{2} \otimes g_{3}=\left({ }^{g_{1}} g_{2} \otimes{ }^{g_{1}} g_{3}\right)\left(g_{1} \otimes g_{2}\right) \text { and } \\
& g_{1} \otimes g_{3} g_{4}=\left({ }^{g_{3}} g_{1} \otimes{ }^{g_{3}} g_{4}\right) \text { for all } g_{1}, g_{2}, g_{3}, g_{4} \in G, \\
& \text { where }{ }^{g_{1}} g_{2}=g_{1} g_{2} g_{1}{ }^{-1} \text { is a left conjugation. By }
\end{aligned}
$$

calculating the nonabelian tensor square of $G$, the standard presentation of the nonabelian tensor square of any group $\mathrm{G}$ can be expressed.

Revised Manuscript Received on June 22, 2019.

Rohaidah Masri, Department of Mathematics, Faculty of Science and Mathematics, UniversitiPendidikan Sultan Idris, 35900TanjungMalim, Perak, Malaysia

Nor Fadzilah Abdul Ladi, Department of Mathematics, Faculty of Science and Mathematics, UniversitiPendidikan Sultan Idris, 35900TanjungMalim, Perak, Malaysia

Nor'ashiqin Mohd Idrus, Department of Mathematics, Faculty of Science and Mathematics, UniversitiPendidikan Sultan Idris, 35900TanjungMalim, Perak, Malaysia

Nor Haniza Sarmin, Department of Mathematical Sciences, Faculty of Science, UniversitiTeknologi Malaysia, 81310 UTM, Johor Bahru, Johor, Malaysia
The nonabelian tensor squares have been introduced by Brown and Loday [1] and is the specialization of the nonabelian tensor product in general. Past researchers have discovered the computations of the nonabelian tensor squares of many sets. For example, Sarminet. al [2], Kappe and Bacon [3] have computed thenonabelian tensor squares of 2-generator nilpotent of class two sets, Blyth, Morse and Moravec [4] have computed the nonabelian tensor squares of free nilpotent sets and Blyth and Morse [5] have created the formula for calculating nonabelian tensor squares of polycyclic sets .

Masri [6] has started the study of the nonabelian tensor squares of Bieberbach sets with certain cyclic spot group in 2009 and focused on Bieberbach sets with cyclic point set of order two. Next, the other studies which are related to the determination of the formula of the nonabelian tensor squares of other Bieberbach sets with different spot groups have also been done by other researchers such as the dihedral set ([7], [8]), the cyclic set of order three and five [9], the symmetric point set $([10,[11])$ and the elementary abelian 2-set point group [12]. The abelian cases for the nonabelian tensor squares of Bieberbach sets can be found in [6] and these findings lead to the generalization of the formula of nonabelian tensor squares of the sets up to dimension $n$. Basedon the generalizations in [6], the homological functors of the Bieberbach set such as the schur multiplier, the nonabelian exterior squares can be computed up to dimension $n$ in [9]. Since the nonabelian tensor square of $S_{2}(3)$ is found to be abelian in [12],this motivates us to study the generalization of the formula of the nonabelian tensor square of this set so that the homological functors of set $S_{2}(3)$ can be determined.

Therefore, the main objective of this paper is to the determine the generalization of the formula of the nonabelian tensor squareof one Bieberbach set of dimension 3 with $C_{2} \times C_{2}$ as the point set, $S_{2}$ (3) up to dimension $n$.Firstly, the polycyclic presentation of $S_{2}(3)$ is given as the following [12] :

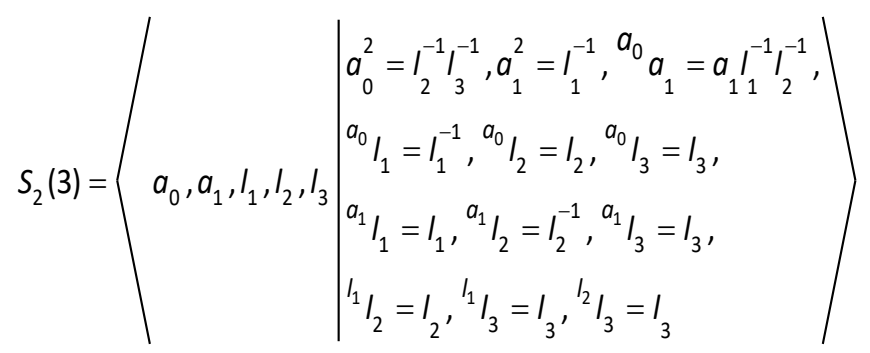




\section{PRELIMINARIES}

Firstly, some structural results related to the computation will be provided in this section. A set $v(G)$ was introduced by Rocco [13] in 1991 and will be used in the computation of the nonabelian tensor square of the set.

Definition 1 [13]

Let $\langle G \mid R\rangle$ be a presentation of set $G$ with relation $R$ and let $G^{\varphi}$ be an isomorphic copy of $G$ via the mapping $\varphi: a \rightarrow a^{\varphi}$ for all $a \in G$. The group $v(G)$ is defined to be

$v(G)=\left\langle G, G^{\varphi} \mid R, R^{\varphi},{ }^{x}\left[a, b^{\varphi}\right]=\left[{ }^{x} a,\left({ }^{x} b\right)^{\varphi}\right]={ }^{x \varphi}\left[a, b^{\varphi}\right], \forall x, a, b \in G\right\rangle$.

The collection of $v(G)$ is related to the nonabelian tensor square of set and can be shown as given in Theorem 1 .

\section{Theorem 1 ([13],[14])}

Let $\sigma$ be a mapping from $G \otimes G$ into $\left[G, G^{\varphi}\right]<v(G)$, for any group $G$ and defined by $\sigma(a \otimes b)=\left[a, b^{\varphi}\right]$ for all $a, b$ in $G$. Then, $\sigma$ is an isomorphism.

Thus, based on Theorem 1, the commutator calculus can be used for the tensor computation within the subgroup of $v$ $(G),\left[G, G^{\varphi}\right]$.

Next, the computational method for polycyclic set developed in [5] is used in order to determine the formula of the nonabelian tensor square of set $S_{2}(3)$. A list of commutator identities in $v(G)$ with left conjugation are given as in the following. Let $a, b$ and $c$ be elements of a group $G$. Then,

$$
\begin{aligned}
& {[a b, c]={ }^{a}[b, c] \cdot[a, c]} \\
& {[a, b c]=[a, b] \cdot{ }^{b}[a, c]} \\
& {\left[a^{-1}, b\right]=\left[a^{-1},[a, b]^{-1}\right] \cdot[a, b]^{-1}} \\
& {\left[a, b^{-1}\right]=\left[b^{-1},[a, b]^{-1}\right] \cdot[a, b]^{-1}} \\
& {\left[a^{-1}, b^{-1}\right]=\left[a^{-1},\left[b^{-1},[a, b]\right]\right] \cdot\left[b^{-1},[a, b]\right] \cdot\left[a^{-1},[a, b]\right] \cdot[a, b]} \\
& { }^{c}[a, b]=\left[{ }^{c} a,{ }^{c} b\right]
\end{aligned}
$$

\section{Definition 2}

Let $G$ be a group. The quotient of group $G$ by its derived subgroup, $G^{\prime}$ is called as the abelianization of group $G$, denoted by $G^{a b}=G / G^{\prime}$.

The next proposition shows the relationship between the structure of the abelianization of $G$ and the central subgroup of the nonabelian tensor square of $G$, denoted by $\nabla(G)$.

\section{Proposition 1 [15]}

Assume that $G$ be a group and $G^{a b}$ is finitely generated. Let $G^{a b}$ is the straight result of the cyclic groups $\left\langle a_{i} G^{\prime}\right\rangle$, for $i=1, \mathrm{~K}, s$ and set $E(G)$ to be $\left\langle\left[a_{i}, a_{j}{ }^{\varphi}\right] \mid i<j\right\rangle\left[G, G^{\prime \varphi}\right]$. Then the following hold:

(i) $\nabla(G)$ is generated by $\left\{\left[a_{i}, a_{i}^{\varphi}\right],\left[a_{i}, a_{j}^{\varphi}\right]\left[a_{j}, a_{i}^{\varphi}\right] \mid 1 \leq i<j \leq s\right\} ;$

$$
\left[G, G^{\varphi}\right]=\nabla(G) E(G) .
$$

\section{Proposition 2 [16]}

Assume that $X, Y$ and $Z$ are abelian groups. Then, the properties of the ordinary tensor product of any two abelian setsare given as in the following.

$$
C_{0} \otimes X \cong X
$$

$$
C_{0} \otimes C_{0} \cong C_{0}
$$

$$
\begin{aligned}
& C_{i} \otimes C_{j} \cong C_{\operatorname{gcd}(i, j)}, \text { for } i, j \in \phi, \text { and } \\
& X \otimes(Y \times Z)=(X \otimes Y) \times(X \otimes Z)
\end{aligned}
$$

Where $C_{0}$ is a cyclic group of infinite order.

\section{Proposition 3 [6]}

Assume that $G$ be any Bieberbach set of dimension $r$ with $P$ as the point group and lattice group $L$. Let $A=G \times F_{s}^{a b}$ where $F_{s}^{a b}$ be a free abelian group of rank $S$. Then $A$ is a Bieberbach group of dimension $r+s$ with point group $P$.

\section{Theorem 2 [17]}

Assume that $G$ be a group. Let $\kappa$ be a commutator mapping from $G \otimes G$ into $G^{\prime}$ and defined by $\kappa(a \otimes b)=[a, b]$, for all $a, b$ in $G$. Here, the kernel of $\kappa$ is in the centre of the nonabelian tensor square of $G$.

Note that $G \otimes G$ is an ordinary tensor square for abelian groups when $G$ is abelian. The nonabelian tensor square of two abelian groups, $X$ and $Y$ is given as follows:

\section{Proposition 4 [17]}

Assume that $G$ be a group such that $G=X \times Y$. Then, $G \otimes G=(X \times Y) \otimes(X \times Y)$ $=(X \otimes X) \times\left(X^{a b} \otimes Y^{a b}\right) \times\left(B^{a b} \otimes Y^{a b}\right) \times(Y \otimes Y)$

Where $X^{a b}=X / X^{\prime}$ and $Y^{a b}=Y / Y^{\prime} \quad$ are the abelianizations of $X$ and $Y$ respectively.

The following proposition gives the derivative subgroup, the abelianization, the central subgroup of the nonabelian tensor square and the nonabelian tensor square of group $S_{2}(3)$

\section{Proposition 5 [18]}

For group $S_{2}(3)$, and $a_{0}, a_{1}, l_{1}, l_{2} \in S_{2}(3)$,

(i) The derived subgroup $S_{2}(3)^{\prime}=\left\langle I_{1}^{-2}, I_{1}^{-1} I_{2}\right\rangle$

(ii) The abelianization

$S_{2}(3)^{a b}=\left\langle a_{0} S_{2}(3)^{\prime}, a_{1} S_{2}(3)^{\prime}\right\rangle \cong C_{0} \times C_{4}$.

\section{Proposition 6 [18]}

Let $a_{0}, a_{1} \in S_{2}(3)$. Then, the central subgroup of the nonabelian tensor square of group $S_{2}(3), \nabla S_{2}(3)$ is given as follows:

$\nabla\left(S_{2}(3)\right)=$ 


$$
\left\langle\left[a_{0}, a_{0}^{\varphi}\right],\left[a_{1}, a_{1}^{\varphi}\right],\left[a_{0}, a_{1}^{\varphi}\right]\left[a_{1}, a_{0}^{\varphi}\right]\right\rangle \cong C_{4} \times C_{8}^{2} \times C_{0} .
$$

\section{Theorem 3 [12]}

The nonabelian tensor square of group $S_{2}(3)$ is found to be abelian and given as follows:

\section{RESULTS}

\section{The Family of Bieberbach Group $S_{2}$ of dimensionn}

By Proposition 3, the family of Bieberbach group $S_{2}$ of dimension $n$ can be defined as follows:

\section{Definition 3}

The group

$$
S_{2}(n)=S_{2}(3) \times F_{n-3}^{a b} \text { for } n \geq 3
$$

is a Bieberbach group with point group $C_{2} \times C_{2}$ of dimension $n$, where $F_{s}^{a b}$ is the free abelian group of rank $s$.

By constructing $S_{2}(n)$ and also by using the normal tensor creation of abelian groups, the formulation of the nonabelian tensor square of $S_{2}$ of dimension $n$ can be determined as given in Theorem 4 .

\section{The calculation of the Formulation of the Nonabelian} Tensor Square of $S_{2}(n)$

Next, Theorem 4 gives the main result of this paper which is the formulation of the nonabelian tensor square of Bieberbach group $S_{2}$ of dimension $n$.

\section{Theorem 4}

For the Bieberbach group of $S_{2}(n)$,

$$
S_{2}(n) \otimes S_{2}(n) \cong C_{2} \times C_{4}{ }^{2 n-5} \times C_{8} \times C_{0}{ }^{n^{2}-4 n+6} \text { For }
$$

$n \geq 4$.

\section{Proof.}

By Definition 3, $S_{2}(n)=S_{2}(3) \times F_{n-3}^{a b}$ for $n \geq 3$. Then by Proposition 4,

$$
\begin{aligned}
& S_{2}(n) \otimes S_{2}(n)=\left(S_{2}(3) \otimes S_{2}(3)\right) \times\left(S_{2}(3) \times F_{n-3}^{a b}\right) \times \\
& \left(F_{n-3}^{a b} \otimes S_{2}(3)^{a b}\right) \times\left(F_{n-3}^{a b} \otimes F_{n-3}^{a b}\right) .
\end{aligned}
$$

By Theorem 3 ,

$$
S_{2}(3) \otimes S_{2}(3) \cong C_{2} \times C_{4} \times C_{8} \times C_{0}^{3} \text {. }
$$

Then, by Proposition 5 (ii), we have $S_{2}(3)^{a b} \cong C_{0} \times C_{4}$. By using Proposition 2(i),

$$
\begin{aligned}
& S_{2}(3)^{a b} \otimes F_{n-3}^{a b} \cong\left(C_{4} \times C_{0}\right) \otimes C_{0}^{n-3} \\
& =\left(C_{4} \otimes C_{0}^{n-3}\right) \times\left(C_{0} \otimes C_{0}^{n-3}\right) \\
& =C_{4}^{n-3} \times C_{0}^{n-3} .
\end{aligned}
$$

And by symmetry,

$$
F_{n-3}^{a b} \otimes S_{2}(3)^{a b}=C_{4}^{n-3} \times C_{0}^{n-3}
$$

Finally, by Proposition 2(ii) we have, $F_{n-3}^{a b} \otimes F_{n-3}^{a b}=C_{0}^{n-3} \times C_{0}^{n-3}$ $\cong C_{0}^{(n-3)^{2}}$.

By collecting terms, then

$S_{2}(n) \otimes S_{2}(n)$

$\cong C_{2} \times C_{4} \times C_{8} \times C_{0}^{3} \times C_{4}^{n-3} \times C_{0}^{n-3} \times C_{4}^{n-3} \times C_{0}^{n-3} \times C_{0}^{(n-3)^{2}}$

$=C_{2} \times C_{4}^{1+(n-3)+(n-3)} \times C_{8} \times C_{0}^{3+(n-3)+(n-3)+(n-3)^{2}}$

$=C_{2} \times C_{4}^{2 n-5} \times C_{8} \times C_{0}^{n^{2}-4 n+6}$

Which completes the proof.

\section{CONCLUSIONS}

The formulation of the nonabelian tensor square of one Bieberbach group is discussed in this paper, $S_{2}(3)$ up to dimension $n$ is developed. This generalization can be used for by other researcher to compute and generalize the other types of homological functors of this group up to dimension $n$.

\section{ACKNOWLEDGEMENT}

The authors would like to express their appreciation to the Ministry of Education (MOE) and UniversitiPendidikan Sultan Idris (UPSI) for sponsored the financial funding through the Fundamental Research Grant Scheme (FRGS) vote no. 2016-0084-102-02. The second author is also indebted to MOE for her My Master Scholarship.

\section{REFERENCES}

1. Brown, R. and Loday, J. L. "Van Kampen Theorems for Diagram of Spaces. " Topology, no.26 (1987):311-335.

2. Sarmin, N. H., Kappe, L. C. and Visscher, M. P. "Two-Generator TwoGroups of Class Two And Their Nonabelian Tensor Squares." Glasgow Mathematical Journal 41, no. 3 (1999):417-430.

3. Bacon, M. R. and Kappe, L. C. "On Capable p-groups of Nilpotency Class Two." Illinois Journal of Mathematics, no. 47 (2003):49-62.

4. Blyth, R. D., Moravec, P. and Morse, R. F. "On the Nonabelian Tensor Squares of Free Nilpotent groups of Finite Rank. "Contemporary Mathematics, no. 470 (2008):27-44.

5. Blyth, R. D. and Morse, R. F. "Computing the nonabelian tensor squares of polycyclic groups." Journal of Algebra, no.321(2009):21392148 .

6. Masri, R. (2009). The Nonabelian Tensor Squares of Certain Bieberbach Groups with Cyclic Point Group of Order Two. Universiti Teknologi Malaysia, Skudai,Malaysia. (PhD Thesis)

7. Mohd Idrus, N. (2011). Bieberbach Groups with Finite Point Groups. Universiti Teknologi Malysia, Skudai, Malaysia. (PhD Thesis)

8. Wan Mohd Fauzi, W. N. F., Mohd Idrus, N., Masri, R. and Tan, Y. T. "On Computing the Nonabelian Tensor Square of Bieberbach Group with Dihedral Point Group of Order 8." Journal of Science and Mathematics Letters, no.2(2014):13-22.

9. Mat Hassim, H. I. (2014). The Homological Functors of Bieberbach Groups with Cyclic Point Groups of Order Two, Three and Five. Universiti Teknologi Malaysia, Skudai, Malaysia. (PhD Thesis)

10. Tan, Y. T., Mohd Idrus, N., Masri, R., Wan Mohd Fauzi, W. N. F., Sarmin, N. H. and Mat Hassim, H. I. "The Nonabelian Tensor Square of Bieberbach Group with Symmetric Point Group of Order Six." Jurnal Teknologi78, no.1 (2016a):189-193.

11. Tan, Y. T., Mohd Idrus, N., Masri, R., Sarmin, N. H. and Mat Hassim, H. I. "On the Generalization of the Nonabelian Tensor Square of Bieberbach Group with Symmetric Point Group." Indian Journal of Science and Technology 9, no. S1 (2016):1-6. 
12. Masri, R., Abdul Ladi, N. F., Mohd Idrus, N., Tan, Y. T. and Sarmin, N. H. "The Nonabelian Tensor Squares of A Bieberbach Group of Dimension Three with Point Group."( International Journal of Advanced and Applied Sciences - In Press).

13. Rocco, N. R. "On a Construction Related to The Nonabelian Tensor Squares of a Group." Bol. Soc. Brasil. Mat.(N. S.) 22, no.1 (1991):6379.

14. Ellis, G. and Leonard, F. "Computing Schur Multipliers and Tensor Products of Finite Groups." Proceedings Royal Irish Academy2, Sect. 95A (1995).

15. Blyth, R. D., Fumagalli, F. and Morigi, M. "Some Structural Results on the Non-abelian Tensor Square of Groups." Journal of Group Theory, no.13 (2010):83-94.

16. Zomorodian, A. J. (2005). Chapter 4 - Topology for Computing. Cambridge University Press, New York, page. 79- 82. 